\title{
A COMPARISON OF COMPUTATIONAL AEROACOUSTIC PREDICTION METHODS FOR TRANSONIC ROTOR NOISE
}

\author{
Kenneth S. Brentner* \\ NASA Langley Research Center, Mail Stop 128 \\ Hampton, VA 23681 \\ Anastasios S. Lyrintzis ${ }^{\dagger}$ and Evangelos K. Koutsavdis ${ }^{\ddagger}$ \\ School of Aeronautics and Astronautics \\ Purdue University, West Lafayette, IN 47907
}

\begin{abstract}
This paper compares two methods for predicting transonic rotor noise for helicopters in hover and forward flight. Both methods rely on a computational fluid dynamics (CFD) solution as input to predict the acoustic near and far fields. For this work, the same full-potential rotor code has been used to compute the CFD solution for both acoustic methods. The first method employs the acoustic analogy as embodied in the Ffowcs Williams-Hawkings ( $\mathrm{FW}-\mathrm{H}$ ) equation, including the quadrupole term. The second method uses a rotating Kirchhoff formulation. Computed results from both methods are compared with one other and with experimental data for both hover and advancing rotor cases. The results are quite good for all cases tested. The sensitivity of both methods to CFD grid resolution and to the choice of the integration surface/volume is investigated. The computational requirements of both methods are comparable; in both cases these requirements are much less than the requirements for the CFD solution.
\end{abstract}

\section{Introduction}

Modern helicopter rotor designs require both high aerodynamic performance and, to an unprecedented degree, low noise radiation. Low noise radiation is particularly important for civilian helicopters, which can only offer significant operational benefits if they can operate in and nearby heavily populated areas. Great progress has been achieved in the accurate and reliable prediction of helicopter rotor noise. A recent review

\footnotetext{
*Research Engineer, Member AHS.

$\dagger$ Associate Professor, Member AHS.

¥Graduate Research Assistant, Student Member AHS. Presented at the American Helicopter Society 52nd Annual FoTum, Washington D.C., June 1996.
}

of helicopter noise prediction is given by Brentner and Farassat [1].

Among the various helicopter rotor noise sources, impulsive noise is particularly annoying as well as difficult to predict accurately. The first type of impulsive noise, high-speed impulsive (HSI) noise, is caused by compressibility effects associated with high-speed blade motion. This HSI noise is generally associated with advancing tip Mach numbers above $M_{A T}=0.85$ and with the appearance of shock waves in the flow field around the rotor. A second type of impulsive noise comes from the interaction of the rotor blades with their vortical wake systems. Blade-vortex interaction (BVI) is a significant source of noise near civilian population centers because it commonly occurs when helicopters are descending for landings. During descent, the advancing tip Mach numbers are usually subcritical. In this paper, we concentrate on HSI noise; however, our methods are readily applicable to BVI as well. We did not consider BVI noise so that we could avoid the errors associated with the wake prediction and, thus, simplify the comparison.

The aerodynamics of HSI noise can be computed accurately with nonlinear computational fluid dynamics (CFD) methods based on full-potential or Euler and Navier-Stokes equations (e.g., [2] and [3]). However, CFD codes are not practical for calculations far (i.e., beyond 2 to 3 rotor radii) from the rotor blades. Thus, near-field CFD solutions have been coupled with more efficient computational methods to propagate the acoustic signals to the far field.

The Lighthill acoustic acoustic analogy [4, 5], as expressed by the Ffowcs Williams-Hawkings ( $\mathrm{FW}-\mathrm{H}$ ) equation [6], has been used for nearly 20 years to predict noise from helicopter rotors. The acoustic analogy predicts discrete-frequency noise quite well, but 
cannot predict HSI noise if the quadrupole term is ignored. Thus, acoustic analogy prediction of HSI noise was forced to wait until CFD methods could predict the transonic flow field around rotor blades moving at high speed.

After advancements in CFD capability enabled accurate computation of the three-dimensional unsteady flow field, another prediction methodology, the Kirchhoff method, became viable as well. The Kirchhoff method assumes that all sound sources are computed correctly by a CFD code and that the acoustic waves are propagated to a surface that surrounds all the sources. When the pressure, the temporal derivative of pressure, and the normal derivative of pressure on the surface are known, the Kirchhoff formulation can determine the acoustic pressure outside the surface via linear wave propagation.

The main purpose of this paper is to compare and contrast these two acoustic prediction methodologies. This comparison is useful because both methods require essentially the same CFD calculation to provide the input data; however, whether one method has an advantage in terms of efficiency, accuracy, and robustness over the other is still unclear. The CFD code used here, as well as both acoustic prediction methods, is implemented in the TiltRotor Aeroacoustic Code (TRAC) [7] at NASA Langley Research Center; we expect that the results of this paper will be useful to future users of this code system.

\section{Acoustic Prediction Methods}

In this section, a brief description of the two acoustic prediction methodologies is given, along with some details of the codes used in this study. The acoustic codes that are compared in this work have similar numerical algorithms and require approximately the same computer resources. The CFD code used in this work will also be discussed.

\section{Acoustic Analogy}

The $\mathrm{FW}-\mathrm{H}$ equation [6] is the most general form of the Lighthill acoustic analogy and is appropriate for predicting the noise generated by the complex motion of helicopter rotors. In differential form, the $\mathrm{FW}-\mathrm{H}$ equation is given by the following inhomogeneous wave equation:

$$
\begin{aligned}
\square^{2} p^{\prime}(\vec{x}, t) & =\frac{\partial}{\partial t}\left[\rho_{o} v_{n} \delta(f)\right]-\frac{\partial}{\partial x_{i}}\left[l_{i} \delta(f)\right] \\
& +\frac{\partial^{2}}{\partial x_{i} \partial x_{j}}\left[T_{i j} H(f)\right]
\end{aligned}
$$

where $p^{\prime}(\vec{x}, t)$ is the acoustic pressure and the three source terms on the right-hand side are known as the thickness, loading, and quadrupole source terms, respectively. The rotor blade is defined by the equation $f=0, v_{n}$ is the local normal velocity of the surface, $l_{i}$ are the components of the local force vector exerted by the surface on the fluid, and $T_{i j}$ is the Lighthill stress tensor. The $\mathrm{FW}-\mathrm{H}$ equation is valid in the entire unbounded space; hence, a formal solution may be obtained by using the free-space Green's function $\delta(g) / 4 \pi r$.

Note in equation (1) that the thickness and loading source terms are surface distributions of sources (indicated by the presence of the Dirac delta function $\delta(f))$. These source terms have been used for several years in rotor noise prediction because they account for most of the acoustic signal when the flow field is not transonic and they do not require knowledge of the field around the blade (although the accurate determination of the blade-surface pressure is still challenging). The quadrupole source, on the other hand, is a volume distribution of sources (indicated by the Heaviside function $H(f))$. The importance of the quadrupole term has long been recognized [8, 9]. However, the quadrupole source has often been neglected in rotor noise prediction because of the computational demands of computing the flow field with sufficient accuracy and integrating over a volume in the acoustic prediction. Recently, Brentner and Holland [10] developed an efficient and robust method to predict HSI noise for a hovering rotor by using a far-field approximation of the quadrupole source. Brentner [11] extended this method for advancing rotors in the code now called WOPWOP+. The WOPWOP+ code is utilized in this work for all acoustic analogy predictions.

\section{WOPWOP+}

The original WOPWOP computer code, developed by Brentner [12], is used to solve the $\mathrm{FW}-\mathrm{H}$ equation for near- and far-field noise. The WOPWOP code uses Farassat's formulation 1A [13], which models only the thickness and loading noise terms in the FW-H equation. The WOPWOP code requires timeaccurate rotor surface pressures and their temporal derivatives for the loading noise prediction. Realistic helicopter blade motions can also be prescribed as inputs. The WOPWOP code is widely used in the rotorcraft community, which includes all major U.S. rotorcraft companies.

A new far-field approximation to the $\mathrm{FW}-\mathrm{H}$ quadrupole source, which is exact for an in-plane farfield observer, has recently been added to the WOPWOP code. (Since HSI noise radiates primarily in the rotor plane, this approximation is also expected to work well for observers outside the rotor plane.) The new version, called WOPWOP+ [10, 11], uti- 
lizes preintegration of the quadrupole volume source in the direction normal to the rotor disk to reduce the computational time required for the quadrupole noise calculation. This far-field approximation leads to quadrupole integrals of the same form as thickness and loading noise (i.e., retarded-time surface integrals). Quadrupole noise prediction is carried out in two parts: a preprocessing stage in which the previously computed flow field is integrated in the direction normal to the rotor disk and a noise computation stage in which quadrupole surface integrals are evaluated for a particular observer position. A retarded-time formulation is used for the quadrupole noise computation; thus, subsonic quadrupole source panel motion is required. The quadrupole noise computation is robust and requires computer resources comparable to those required for thickness and loading noise prediction.

\section{Kirchhoff Formulation}

The second prediction method for far-field helicopter acoustics is based on Kirchhoff's theorem. Pressures and pressure derivatives from the near-field CFD computation are utilized on a surface that encloses the rotor blades. The derivation of the Kirchhoff formulation depends on the assumption that only linear acoustic propagation occurs outside the Kirchhoff integration surface and all acoustic sources and nonlinear effects are contained within the surface. Hence, the sound speed is constant for the acoustic wave propagation from the Kirchhoff surface to the far field. The most important nonlinearities are captured, however, because the CFD solver computes the complete nonlinear acoustic field inside the Kirchhoff surface. The method was first demonstrated in rotorcraft aeroacoustics for two-dimensional cases and was later applied in three-dimensional cases. A general review of the use of the Kirchhoff method in computational acoustics is given by Lyrintzis [14].

Farassat and Myers [15] have derived a general Kirchhoff formula that gives the pressure signal of an observer in the stationary coordinate system as a function of the surface integral over surface $S$ of the pressure, the normal derivative of the pressure, and the time derivative of the pressure, where surface $S$ is moving at an arbitrary speed. The formulation was derived for a deformable surface; however we use the rigid-surface version as follows:

$$
p\left(\mathbf{X}_{*}, t_{*}\right)=\frac{1}{4 \pi} \iint_{S}\left[\frac{E_{1}}{r\left(1-M_{r}\right)}+\frac{E_{2} p}{r^{2}\left(1-M_{r}\right)}\right]_{\tau^{*}} d S
$$

where

$$
\begin{aligned}
E_{1} & =\left(M_{n}^{2}-1\right) p_{n}+M_{n} M_{t} \cdot \nabla_{2} p-a_{\infty}^{-1} M_{n} \dot{p} \\
& +\frac{a_{\infty}^{-1}}{\left(1-M_{r}\right)}\left[\left(\dot{n}_{r}-\dot{M}_{n}-\dot{n}_{M}\right) p+\left(\cos \theta-M_{n}\right) \dot{p}\right] \\
& +\frac{a_{\infty}^{-1}}{\left(1-M_{r}\right)^{2}}\left[\dot{M}_{r}\left(\cos \theta-M_{n}\right) p\right] \\
E_{2} & =\frac{1-M^{2}}{\left(1-M_{r}\right)^{2}}\left(\cos \theta-M_{n}\right)
\end{aligned}
$$

The dot over $M$ and $n$ denotes the source time $\tau^{*}$ derivative. Here, $\left(\mathbf{X}_{*}, t_{*}\right)$ and $(\mathbf{X}, \tau)$ represent the observer position and observer time, and the source position and source time, respectively. The subscript $\tau^{*}$ in equation (2) indicates that the integrand is evaluated at the source emission time $\tau^{*}$, which is the solution of the equation

$$
\tau-t_{*}+\left|\mathbf{X}_{*}-\mathbf{X}(\tau)\right| / a_{\infty}=0
$$

In addition, the following definitions are introduced:

$$
\begin{aligned}
\dot{M}_{r} & =\dot{\mathbf{M}} \cdot \hat{\mathbf{r}}, & \dot{M}_{n}=\dot{\mathbf{M}} \cdot \mathbf{n}, \\
\dot{n}_{r} & =\dot{\mathbf{n}} \cdot \hat{\mathbf{r}}, & \dot{n}_{M}=\dot{\mathbf{n}} \cdot \mathbf{M}
\end{aligned}
$$

The formulation in equation (2) is valid when the observer is stationary and the surface is moving with arbitrary velocity. The case in which the observer is moving along with the rotor (e.g., rotor in a wind tunnel with flow) can also be modeled simply by moving the observer position for each point in the time history. A limitation of the formula is that singularities appear for supersonically moving surfaces. Farassat and Myers [16] have derived a new formula to avoid this problem; however the new formula has not yet been applied in practical problems.

Two approaches have been used to implement the Kirchhoff integral for three-dimensional rotorcraft noise prediction. They differ as to whether or not the Kirchhoff surface rotates with the helicopter blades. The first approach (e.g., [17] and [18]) employs a rotating Kirchhoff surface. This approach has an advantage in that the Kirchhoff integrals use the same computational mesh as the CFD calculations; therefore, interpolations onto the Kirchhoff surface are not required. One disadvantage of the rotating Kirchhoff implementation, however, is that parts of the surface sufficiently far from the center of rotation will potentially have supersonic velocity. The requirement for subsonic source motion imposes a limitation on the radial extent of the Kirchhoff surface for rotors whose tip Mach numbers approach unity.

The second implementation approach (e.g., [19] and [20]) employs a nonrotating Kirchhoff surface that 
completely surrounds the spinning rotor blades. Interpolation of the input data is required to transfer the rotating CFD solution to the nonrotating Kirchhoff surface; however, because the surface only translates with the rotor, sonic velocities are avoided. Recently, Strawn et al. [21] compared the rotating and nonrotating Kirchhoff formulations for both HSI and BVI noise. Both methods yielded similar results for the cases considered. Note that high-accuracy CFD information (usually generated by an Euler code) out to the nonrotating Kirchhoff surface is needed. This requirement could increase the $\mathrm{CFD}$ needs of the nonrotating method. In this paper, the rotating Kirchhoff implementation is used because the full-potential solver computes the solution only relatively close to the blade. In addition, the algorithmic details of the rotating Kirchhoff method are quite similar to those of the the acoustic analogy code.

\section{$R K I R$}

The numerical implementation of the Kirchhoff formula of Farassat and Myers for a rotating surface, known as RKIR, is described in reference [17]. The RKIR code requires an external source of data for pressure and pressure derivatives on a surface. In addition to the time derivatives of pressure, the Kirchhoff scheme also requires spatial gradients of pressure. The computation of the temporal and spatial derivatives of the pressure are performed as a subroutine in the CFD flow solver. The Kirchhoff surface is typically located 1 to 2 chord lengths away from the rotor surface and surrounds the rotor blades. The surface has the appearance of a cylinder with a tip-end cap. The root end of the Kirchhoff surface is not included because its contribution is negligible. The Kirchhoff surface must be chosen to have only subsonic source motion for the reasons described previously. This restriction is the same as in the WOPWOP+ code. The RKIR code was recently modified [22] to include the same blade motions as WOPWOP (i.e., pitching, lead lag, and feathering) and to make the input and output of RKIR as close to that of WOPWOP as possible. This version, which is part of the NASA Langley Research Center TRAC system [7], was used in this study.

\section{CFD Model}

A version of the full-potential rotor code FPR [2] was used to provide the flow-field computation in this work because it provides a fast and accurate CFD solution, is widely accepted by the U.S. rotorcraft industry, and has recently been coupled to both acoustic codes exercised in this work. The FPR code uses a finite-difference scheme to compute three-dimensional unsteady transonic flows around the rotor blades. This code is fast in comparison with solutions of the Euler and Navier-Stokes equations and still gives a realistic representation of the physical flow field. The fullpotential assumptions of inviscid and irrotational flow are appropriate for a transonic rotor. The flow field is dominated by inviscid effects, and the shocks are not strong enough to generate significant vorticity.

The code was extended to accept wake information to account properly for rotor inflow and blade-vortex interactions in the version known as FPRBVI [23]. This version was used in the present study, although the wake and BVI capabilities were not utilized. All flow-field calculations computed here are for nonlifting rotors. The FPRBVI code has an internal algebraic grid generator that is different from that in the original FPR code. This internal grid generator was used for all cases presented here. The near-field solutions from FPRBVI should provide accurate information for far-field calculations with both acoustic methods. The same FPRBVI solution was used as input to both WOPWOP+ and RKIR.

\section{Results}

Acoustic predictions were made for three different model rotors operating both in hover and forwardflight test conditions. Two different grids were used in the FPRBVI computations. The first grid was a standard $O$ grid with 80 points in the wrap-around direction, 36 points in the spanwise direction ( 27 points on the blade), and 24 points in the direction normal to the blade surface. A second, finer grid was also used, which had 150 points in the wrap-around direction, 50 points in the spanwise direction (34 points on the blade), and 50 points in the normal direction. The computations were performed by first running FPRBVI in a quasi-steady mode for 400 time steps and then continuing in an unsteady mode. The unsteady solution was superior to the quasi-steady calculation for the hover cases, so this solution was used for both the hover and forward-flight calculations. The acoustic codes, however, only used the CFD solution from one time step for the hover predictions. For the standard grid, an unsteady time step of $0.125^{\circ}$ azimuth was used; for the fine grid, a smaller time step of $0.0625^{\circ}$ azimuth was required. The standard-grid computation required approximately $0.5 \mathrm{hr}$ on a Cray C90, whereas the fine grid required approximately $5 \mathrm{hr}$.

A comparison of computation time required by the acoustic codes is somewhat tentative because both of these codes are relatively immature. Nevertheless, we can compare of the computational resources needed by the current version of each code. The present codes depend upon file input/output ( $/$ /O) for data storage and manipulation during the prediction. File I/O can be as much as $25 \%$ or more of the total computational time 
required for the acoustic predictions. For the case of a hovering rotor with the standard CFD grid, both codes require nominally $70 \mathrm{CPU}$ sec per observer to run on an HP 9000 Series 735-99 workstation. For the fine grid, the WOPWOP+ code requires approximately 8 seconds more while the RKIR code needed an additional 39 seconds. The RKIR code required more time because the control surface for the fine grid contained nearly twice as many points; however, the source grid generated by the WOPWOP+ preprocessor did not change in size. For forward-flight computations with the standard grid, WOPWOP+ required $791 \mathrm{sec}$ to run (691 sec for the preprocessor and approximately 100 sec for the acoustic computation) while the RKIR code only needed 520 sec. Much of the time expended by the WOPWOP+ preprocessor was associated with file $\mathrm{I} / \mathrm{O}$. The preprocessor is only run once per operating condition; thus, if calculations are made for more that one observer location the WOPWOP+ computation is faster. The processing time required to compute the temporal and spatial derivatives of pressure in the FPRBVI code is not accounted for in the Kirchhoff times. It is important to note that the computational time necessary to perform the acoustic predictions was substantially less than that required to compute the flow field.

For the Kirchhoff predictions, a choice of the Kirchhoff surface location is required. Several surface locations, which correspond to grid lines, were evaluated in this work. Two surfaces, identified as the "large cylinder" and the "small cylinder," respectively, are presented in this paper. For the standard grid, the large cylinder corresponds to the 21st grid line normal to the blade surface; the small cylinder corresponds to the 18th grid line normal to the blade surface. The tip-end cap for both surfaces was located on the 31st spanwise grid plane. The location of these surfaces, shown for a standard grid in Fig. 1, was nominally maintained for all grid and rotor-blade combinations. The volume integration in WOPWOP+ used approximately the same volume contained by the Kirchhoff surfaces in Fig. 1.

\section{Sensitivity Analysis}

To determine the sensitivity and robustness of each prediction method, comparisons were made between the size of the volume around the rotor blade (i.e., the control surface position in the case of the Kirchhoff code) and the resolution of the CFD grid. These comparisons were made for most of the cases presented in this paper; however, for brevity we present only the results for one rotor and operating condition.

The first comparison was for a hovering rotor operating at a high tip speed. The results of an experiment

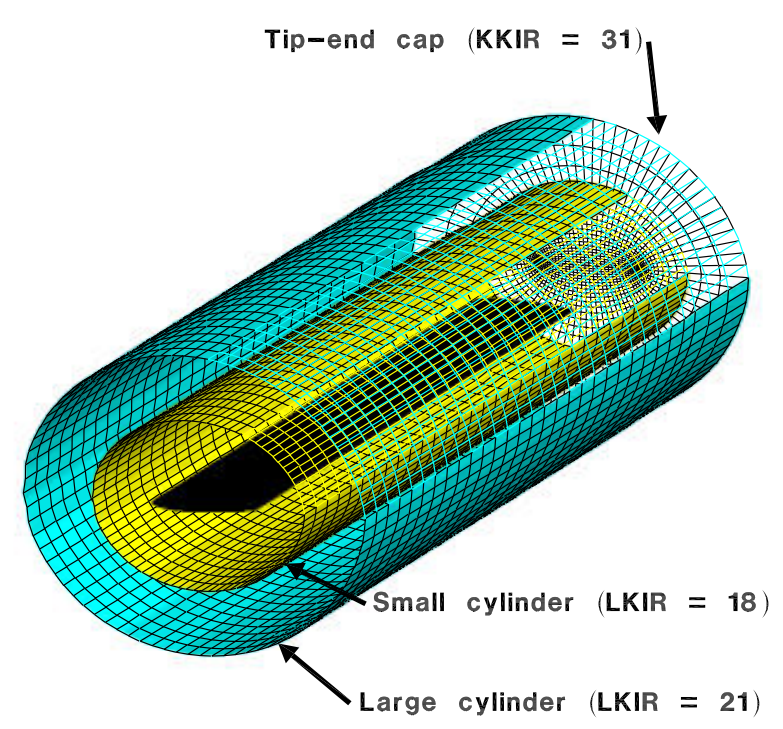

Figure 1. Location of the Kirchhoff surfaces (standard UH-1H grid).

conducted by Purcell [24] are used for comparison. The rotor is a one-seventh scale UH-1H model main rotor with straight, untwisted blades and an NACA 0012 airfoil section. The rotor radius is $1.045 \mathrm{~m}$ with a chord of $7.62 \mathrm{~cm}$; thus, the blade aspect ratio is 13.7. The test was conducted with very little lift produced by the rotor. Comparisons with predicted and measured acoustic signals are made for an in-plane microphone located 3.09 rotor radii from the rotor hub. The experiment was repeated for several Mach numbers. Three hover Mach numbers, $M_{H}=0.70,0.88$, and 0.90 , are used in this paper to compare the predicted and measured results.

The hover case with $M_{H}=0.88$ was chosen for the sensitivity comparison because the flow field is supercritical but not delocalized. This case should test the two acoustic codes because transonic effects are strong; however, this case still falls within the range of validity of WOPWOP+ and RKIR because supersonic source motion is not expected to be required. In Figs. 2 and 3 , a comparison between the predictions with the standard and the fine grid in the FPRBVI computation is presented. The large cylinder was used. The experimental data are also shown in the figures for reference. Although the fine-grid inputs yield slightly better results (the difference is approximately 3 percent), the computing cost for the fine-grid solution with FPRBVI is substantially greater. Both codes predict the magnitude of the negative pressure peak with approximately the same accuracy. The agreement with the data is 


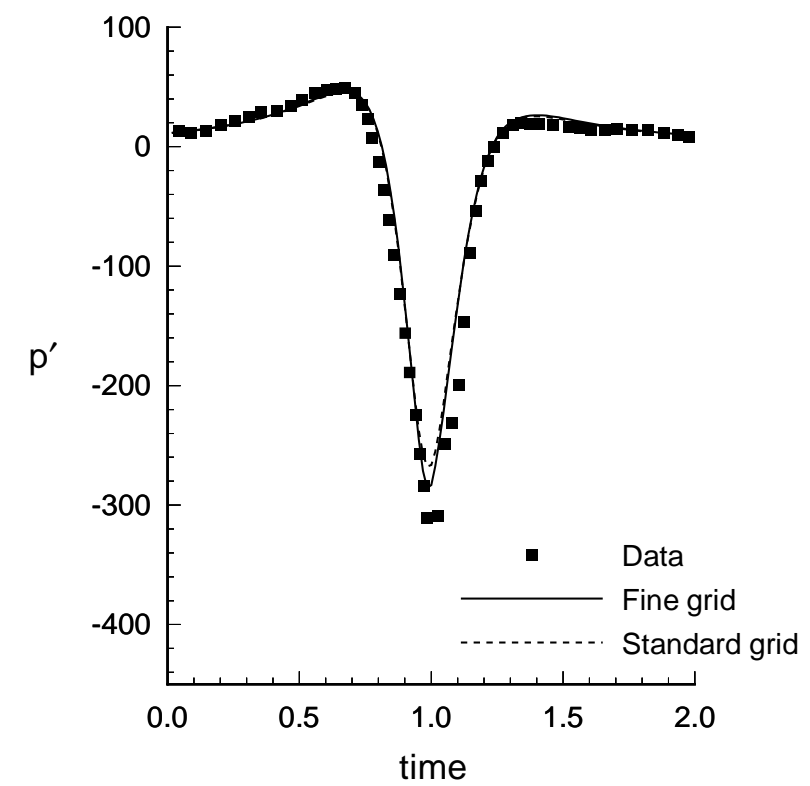

Figure 2. Comparison of standard- and fine-grid FPRBVI inputs to WOPWOP+ (large cylinder) for UH-1H model rotor operation at $M_{H}=0.88$.

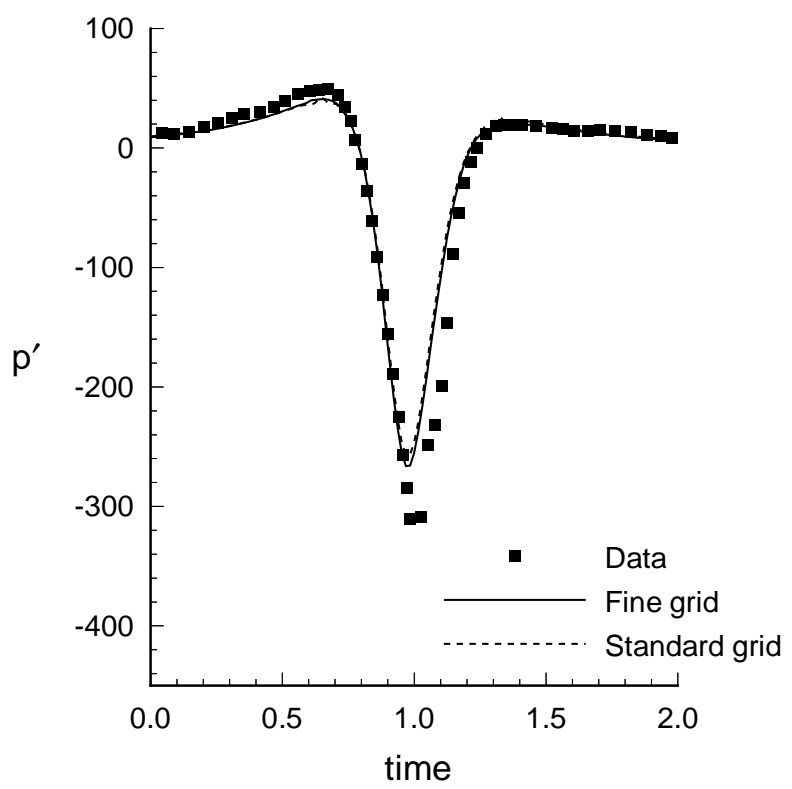

Figure 3. Comparison of standard- and fine-grid FPRBVI inputs to RKIR (large cylinder) for UH-1H model rotor operation at $M_{H}=0.88$.

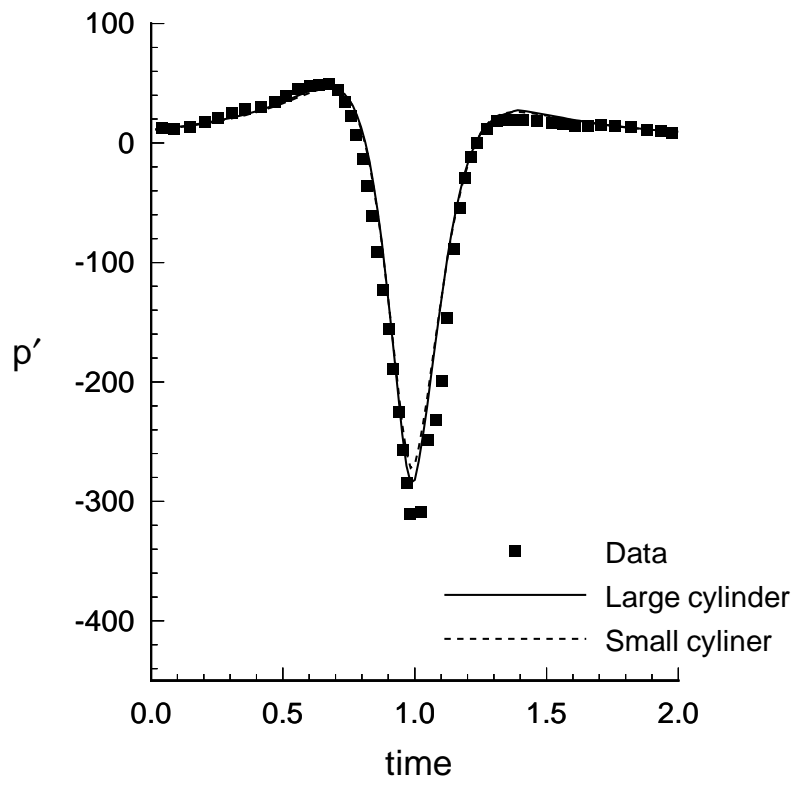

Figure 4. Comparison of small- and large-cylinder inputs to WOPWOP+ (fine grid) for UH-1H model rotor operation at $M_{H}=0.88$.

quite good for both methods.

In Figs. 4 and 5, the acoustic predictions made by using the two surfaces shown in Fig. 1 are compared. The fine-grid results were utilized for this comparison because the fine grid yields slightly better agreement with the data. The tip-end surface was chosen to be just inside the sonic cylinder for this Mach number. The results were relatively insensitive to the choice of the position of this end surface, as long as it was located near the sonic cylinder for high-speed cases. In Fig. 4, the WOPWOP+ calculation indicated nearly no change in predicted signal due to the increase in integration volume. The reason that the predicted signal does not change is apparently because the main quadrupole contributions come primarily from the region near the blade. The slight change in the negative peak pressure when the larger volume is used corresponds to the inclusion of more quadrupole sources. The results for the Kirchhoff method in Fig. 5, however, show a large sensitivity to the location of the Kirchhoff surface. These results show a nearly constant shift in the curves; this shift is nearly identical for both the standard and the fine grid. One possible explanation for this shift is the nonlinearity of the input signal. Another explanation may be that the spatial pressure derivatives are not computed with sufficient accuracy on either grid. An important goal of future work will be the determination of the cause of this variability. 


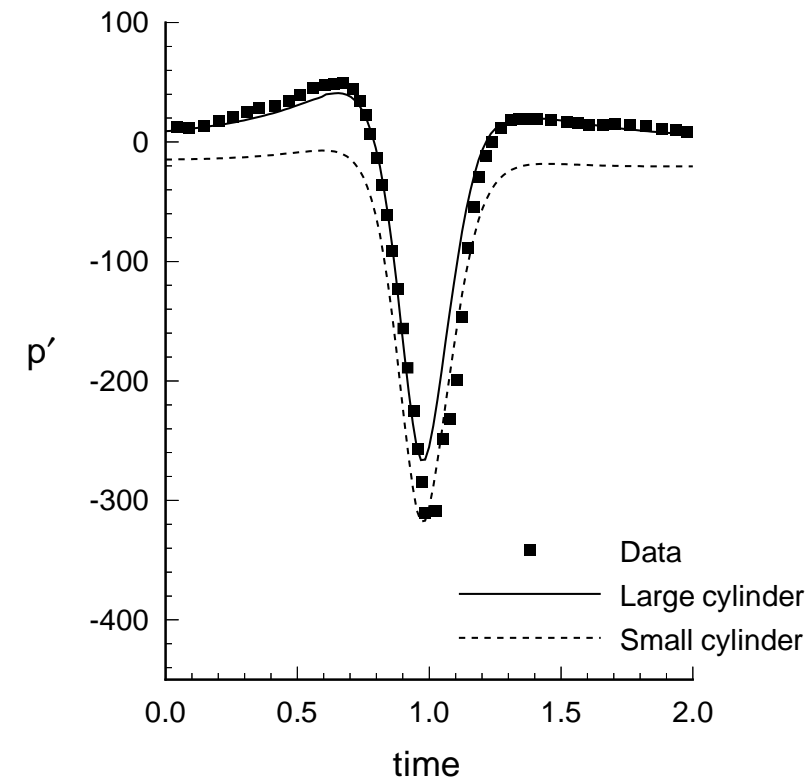

Figure 5. Comparison of small- and large-cylinder inputs to RKIR (fine grid) for UH-1H model rotor operating at $M_{H}=0.88$.

Now that we have examined the sensitivity of the codes for a representative case, we turn our attention to comparison of method performance for a range of operating conditions. For the following cases, we present only predictions from the large cylinder on the standard CFD grid. These comparisons are representative of computations that can be routinely made with the FPRBVI solver.

\section{Hover Results}

For $M_{H}=0.70$, the flow field around the rotor is subcritical; thus, the quadrupole contribution in the WOPWOP+ solution is not expected to be very large. Figure 6 shows the WOPWOP+ and RKIR predictions of the acoustic pressure compared with the Euler calculation (TURNS code [3]) of Baeder et al. [19]. The quadrupole component of the total signal predicted with WOPWOP+ is also shown in Fig. 6. Notice that although the quadrupole contribution is small, the contribution is significant even for this low subcritical Mach number and should be included in an accurate acoustic prediction when the acoustic analogy method is used. The Kirchhoff prediction is nearly the same as the WOPWOP+ prediction. The excellent agreement between the three predictions suggest that the FPRBVI solution is accurate. (The acoustic analogy signal is dominated by thickness noise which is computed from a knowledge of only the blade kinematics and geometry. Thus if the FPRBVI solu-

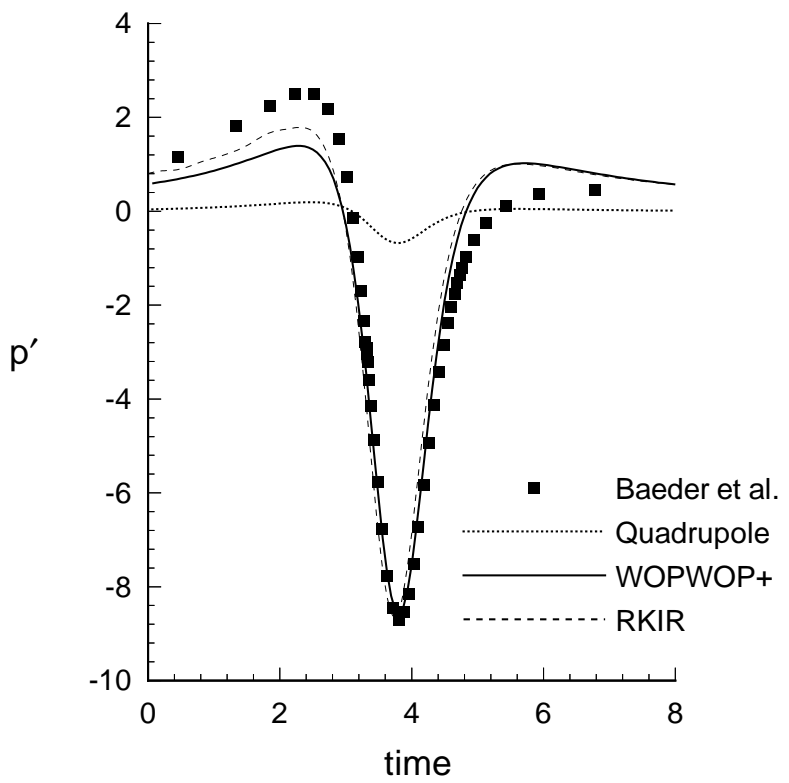

Figure 6. Comparison of acoustic predictions for UH$1 \mathrm{H}$ model rotor operation at $M_{H}=0.70$ with the standard FPRBVI grid. (Reference computation from Baeder et al. [19].)

tion is not sufficiently accurate at this Mach number, it will have only a small effect on the acoustic analogy prediction, but the Kirchhoff solution will suffer.)

The case for which $M_{H}=0.88$ has already been examined, but we would like to show a direct comparison of the WOPWOP+ and RKIR predictions. (See Fig. 7.) In this case, the quadrupole component of the WOPWOP+ prediction is a much higher percentage of the total (nearly 50 percent) than for the cases in which $M_{H}=0.70$. The Kirchhoff prediction is nearly indistinguishable from the WOPWOP+ prediction. The slight underprediction of the negative peak, as compared to the experimental data, is at least partially an indication that the grid resolution of the standard grid is only marginally sufficient. This inadequacy of the standard grid was also evident in the earlier comparison of the standard- and fine-grid results.

For $M=0.90$, the flow becomes delocalized (i.e. the supersonic region on the blade surface extends to a region in which the free stream is moving supersonically relative to the rotor-blade tip [8]). When delocalization occurs, shock waves on the rotor surface are allowed to propagate to the far field and the radiated noise dramatically increases. Because both methods are currently limited to subsonically moving sources, the accuracy of the results was expected to degrade in this case. Figure 8 shows the results for 


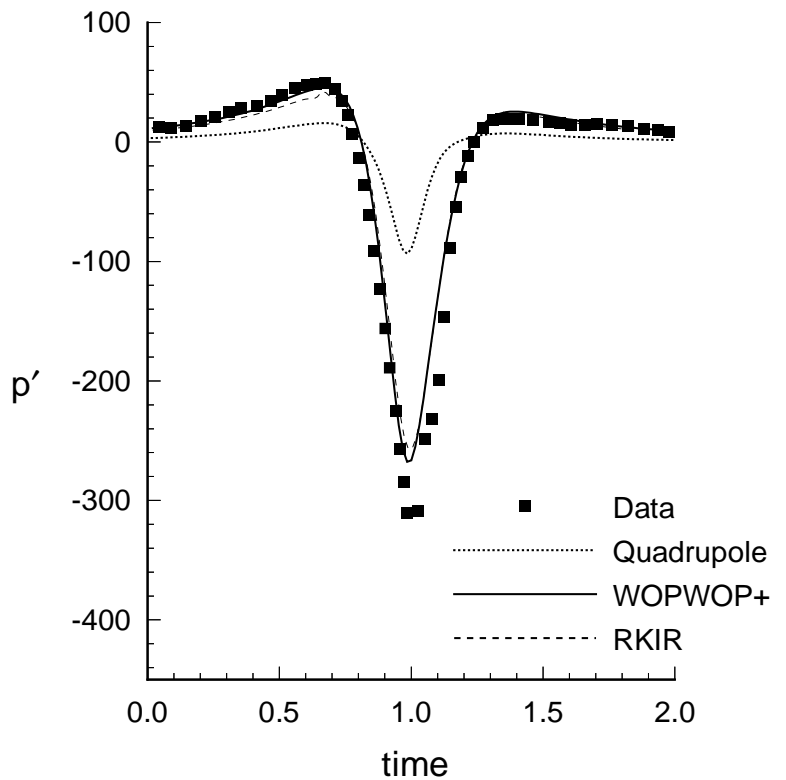

Figure 7. Comparison of acoustic predictions for $\mathrm{UH}-$ $1 \mathrm{H}$ model rotor operation at $M_{H}=0.88$.

$M_{H}=0.90$ with the standard grid. For this case, the quadrupole component is the largest component of the noise. Notice the dramatic increase in the peak negative pressure and the sawtooth waveform shape that results with a very small increase in Mach number; these changes are characteristics of delocalization. The WOPWOP+ and RKIR predictions for this condition are again in close agreement. Both codes fail to fully predict the sharp nonlinear steepening of the wave pulse. The negative peak the quadrupole contribution has the shape of the measured signal; however, the quadrupole component is not large enough. This underprediction results from not including the acoustic sources that are moving supersonically.

\section{Advancing Rotor Results}

Two advancing rotor cases, chosen from separate experiments, are now described in this section.

\section{OLS Rotor}

The first comparison for an advancing rotor is made for the Operational Loads Survey (OLS) model rotor. This rotor is a one-seventh scale model of the main rotor of the AH-1 series helicopter. Model rotor acoustic data were obtained by Schmitz et al. [25] in the DuitsNederlandse Windtunnel (DNW) for several high forward speeds. Several microphones were used in the test, but we will use data from three in-plane positions located 1.72 rotor diameters from the rotor hub ahead of the rotor. The three microphones selected for use were in the following positions: $30^{\circ}$ on the ad-

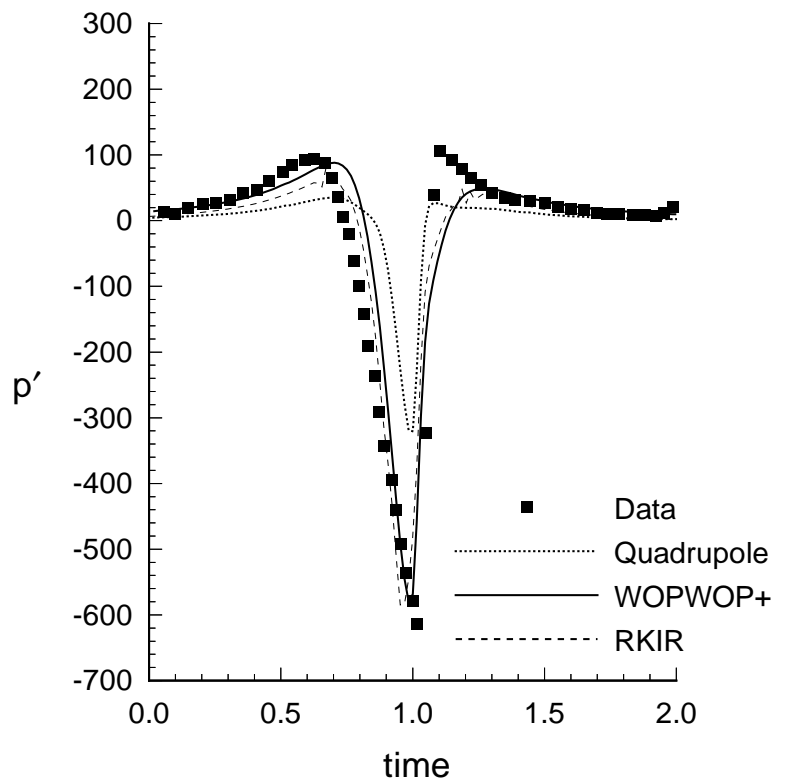

Figure 8. Comparison of acoustic predictions for $\mathrm{UH}-$ $1 \mathrm{H}$ model rotor operation at $M_{H}=0.90$.

vancing side of the rotor, on the centerline, and $30^{\circ}$ on the retreating side of the rotor. The OLS rotor was a two-bladed rotor. For the following comparison, the rotor was operating with an advancing tip Mach number $M_{A T}=0.84$ and an advance ratio $\mu=0.27$. A complete description of the test can be found in Ref. [26].

Figures 9-11 show the results for this case at the advancing-side, centerline, and retreating-side microphone positions, respectively. In all cases, we see that the results from both codes compare well with the experimental measurements. The Kirchhoff results are closer to experimental data for all three microphone positions. Because peak negative pressure for this condition is not large, the inclusion of loading in the predictions may improve the waveform shape.

\section{UTC Four-bladed rotor}

The second advancing rotor case is for a four-bladed United Technologies Corporation contemporary design model rotor. This rotor was pressure instrumented and tested in the DNW tunnel. The rotor had swept tips and modern airfoil sections. The test is described by Yu et al. [27]; the acoustics portion of the test is summarized by Liu and Marcolini [28]. The measurements used in our comparison were taken in the tip-path plane of the rotor, at $30^{\circ}$ on the advancing side, on the centerline, and at $30^{\circ}$ on the retreating side ahead of the rotor, 1.5 diameters from the rotor hub. Comparisons between the predicted and experimental data 


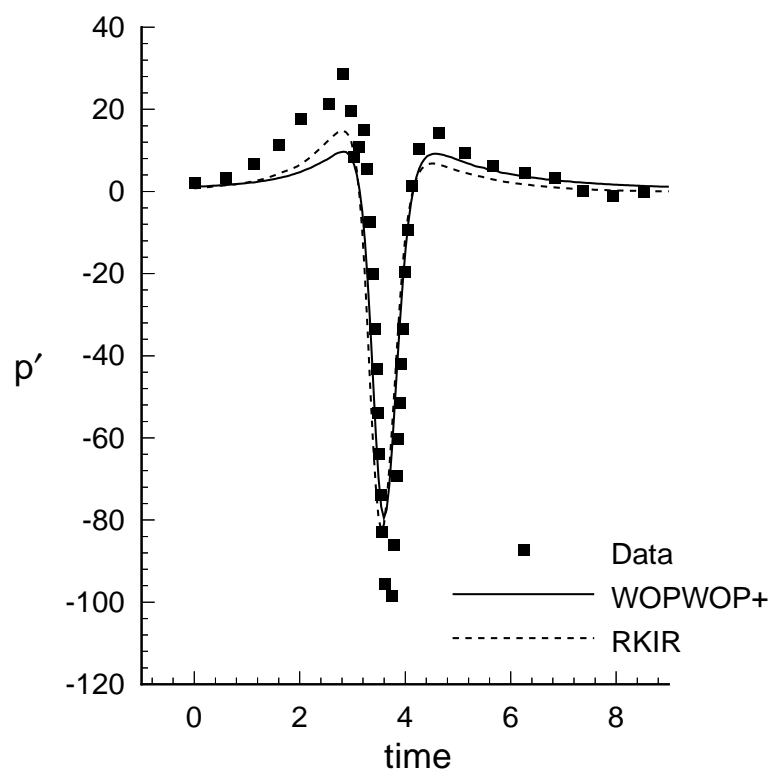

Figure 9. Comparison of advancing-side microphone predictions (standard grid) for OLS model rotor operation at $M_{A T}=0.84$, and $\mu=0.27$.

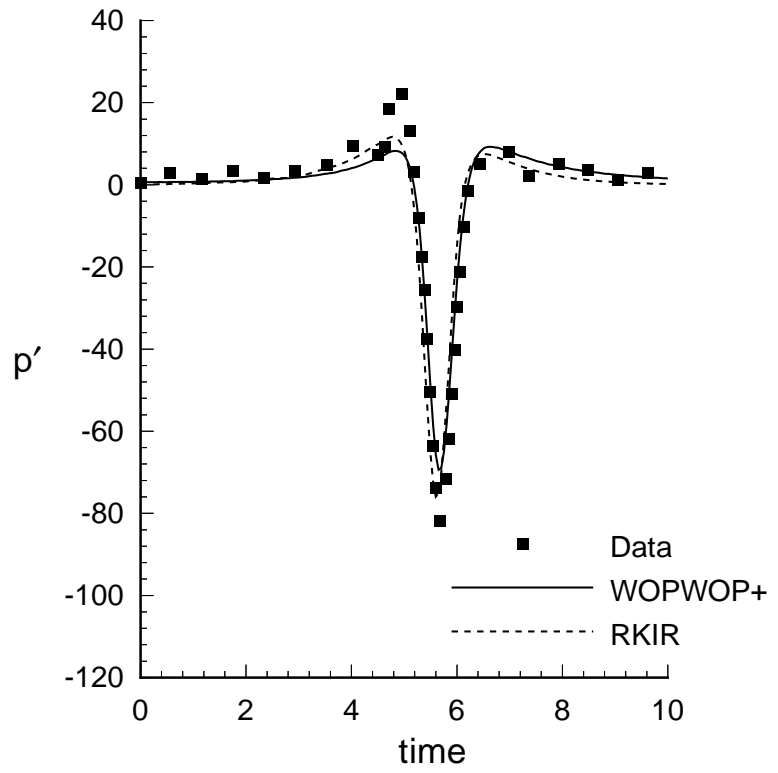

Figure 10. Comparison of centerline microphone predictions (standard grid) for OLS model rotor operation at $M_{A T}=0.84$, and $\mu=0.27$.

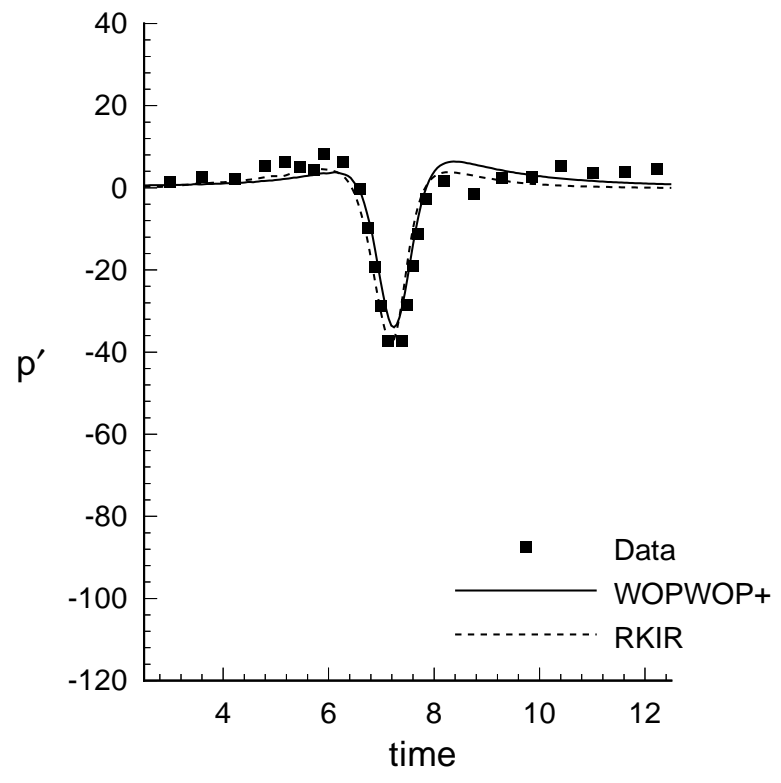

Figure 11. Comparison of retreating-side microphone predictions (standard grid) for OLS model rotor operation at $M_{A T}=0.84$, and $\mu=0.27$.

were made for a high-speed condition $M_{A T}=0.94$, and $\mu=0.32$. The flow around the rotor was delocalized. A degraded accuracy was expected for this case because neither code accounts for the supersonically moving sources; however, this case was expected to provide a good indication of code robustness.

Figures 12-14 show the results for this case at each microphone location. The quality of the results overall is good. Both codes predict lower values for the peak pressures than those measured experimentally, which can be attributed to the absence of the supersonically moving sources. Nevertheless, the predictions are significantly better than would have been obtained by the WOPWOP code, which includes only thickness and loading sources. Note that the RKIR prediction is better for the retreating-side microphone, whereas the WOPWOP+ prediction is better for the advancingside microphone. The reason for this difference is unclear. The Kirchhoff prediction does not agree well with either WOPWOP+ or the data away from the negative peak; this may indicate that the placement of the Kirchhoff surface is not optimal.

\section{Concluding Remarks}

Two methods were compared for computing rotorcraft impulsive noise: an acoustic analogy approach that includes the Ffowcs Williams-Hawkings quadrupole terms and a rotating Kirchhoff method. 


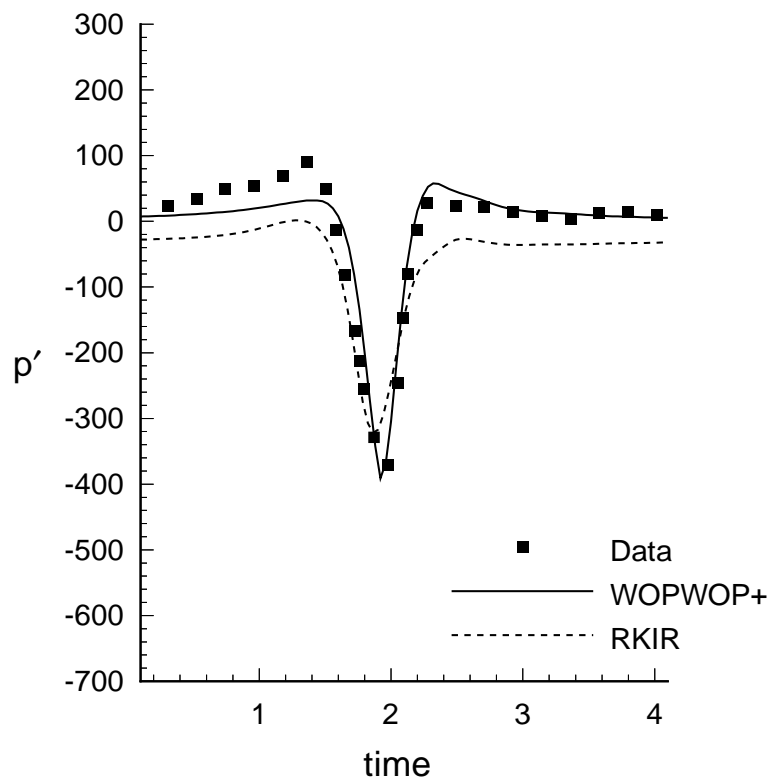

Figure 12. Comparison of advancing-side microphone predictions (standard grid) for UTC model rotor operation at $M_{A T}=0.93$, and $\mu=0.32$.

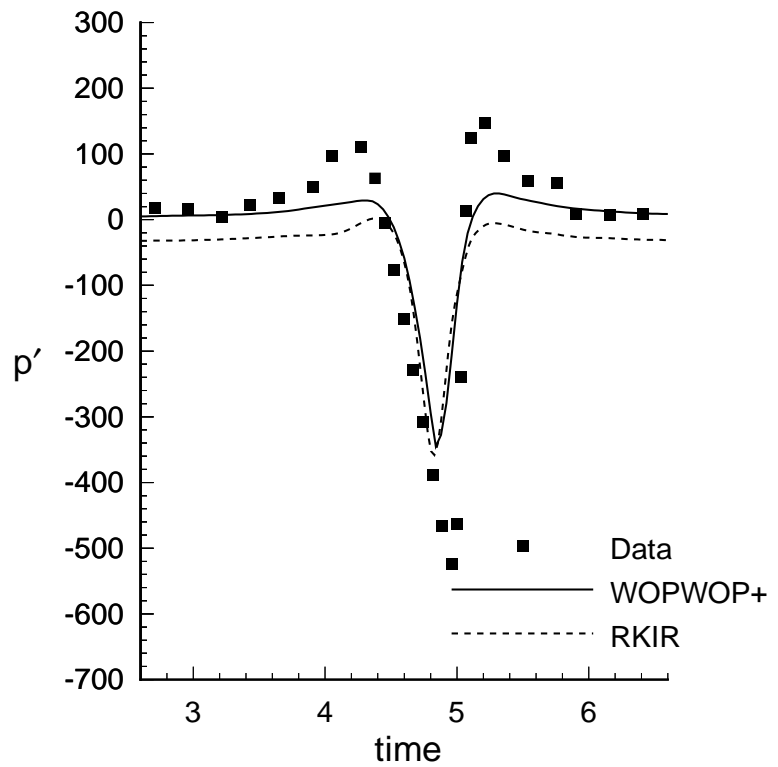

Figure 13. Comparison of centerline microphone predictions (standard grid) for UTC model rotor operation at $M_{A T}=0.93$, and $\mu=0.32$.

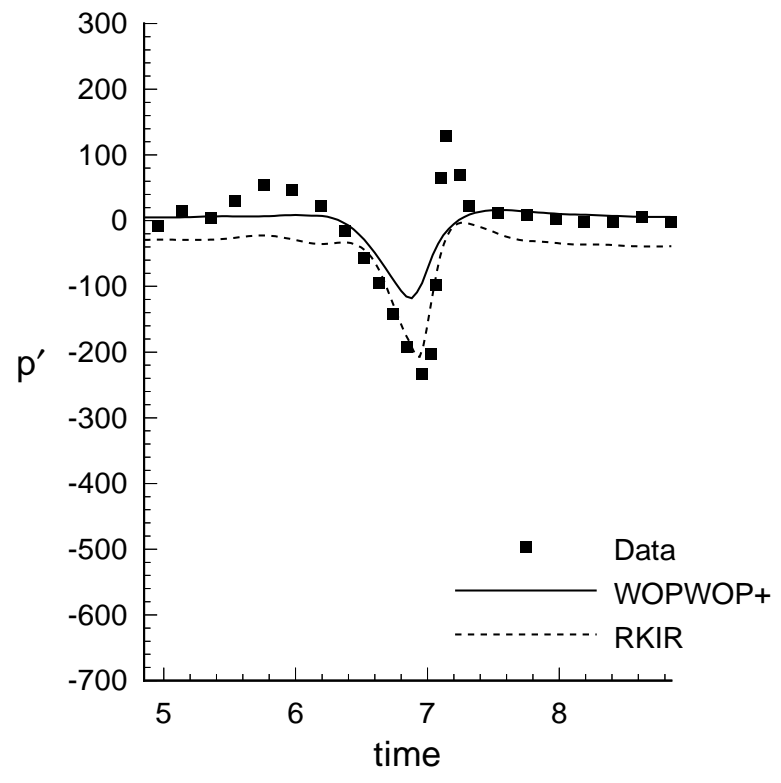

Figure 14. Comparison of retreating-side microphone predictions (standard grid) for UTC model rotor operation at $M_{A T}=0.93$, and $\mu=0.32$.

Both methods rely on computational fluid dynamics input. Because the CFD code and both acoustic prediction codes are implemented in the TiltRotor Aeroacoustic Code (TRAC) at NASA Langley Research Center, the results in this paper should be useful to future users of this code system. Although the methods were coupled to a full-potential rotor finitedifference method, these methods can easily be coupled to other CFD solvers, as well.

Both methods agree well with the experimental measurements for a variety of rotors and transonic operating conditions below the delocalization Mach number. The Kirchhoff method is more sensitive to the choice of control surface; however, the choice of the Kirchhoff surface is nearly independent of the Mach number in the cases considered here. Because neither code includes supersonically moving sources, performance is degraded for delocalized cases. For both codes, results with a fine grid are more accurate than the results with a standard grid, but the accuracy improvements are not dramatic. The computer time required by the flow solver, however, is substantially greater for the fine grid.

The computational resources required by both computer codes is roughly equivalent. The Kirchhoff code requires less computer time for a single observer than WOPWOP+ in forward-flight cases, but the time to compute hover predictions is approximately the same. The computational requirements of the two acoustics 
prediction methods are minimal, however, in comparison with the resources required by the CFD solver.

\section{Acknowledgments}

The author's would like to acknowledge that the Euler calculation for the $\mathrm{UH}-1 \mathrm{H}$ rotor case for which $M_{H}=0.70$ was obtained from Professor James D. Baeder, University of Maryland. The OLS data was provided by Mr. Klaus J. Schultz, DLR. Some calculations for this paper were performed at the computational facilities of the Pittsburgh Supercomputing Center.

\section{References}

[1] Brentner, K. S., and Farassat, F., "Helicopter Noise Prediction: The Current Status and Future Direction," Journal of Sound and Vibration, Vol. 170, No. 1, 1994, pp. 79-96.

[2] Strawn, R. C., and Caradonna, F. X., "Conservative Full-Potential Model for Unsteady Transonic Rotor Flows," AIAA Journal, Vol. 25, No. 2, Feb. 1987, pp. 193-198.

[3] Srinivasan, G. R., and Baeder, J. D., "TURNS: A Free-Wake Euler/Navier-Stokes Numerical Method for Helicopter Rotors," AIAA Journal, Vol. 31, No. 5, May 1993, pp. 959-962.

[4] Lighthill, M. J., "On Sound Generated Aerodynamically. I: General Theory," Proceedings of the Royal Society, Vol. A221, 1952, pp. 564-587.

[5] Lighthill, M. J., "On Sound Generated Aerodynamically. II: Turbulence as a Source of Sound," Proceedings of the Royal Society, Vol. A222, 1954, pp. 1-32.

[6] Ffowcs Williams, J. E., and Hawkings, D. L., "Sound Generated by Turbulence and Surfaces in Arbitrary Motion," Philosophical Transactions of the Royal Society, Vol. A264, 1969, pp. 321-342.

[7] Burley, C. L., Marcolini, M. A., Brand, A. G., and Conner, D. A., "TiltRotor Aeroacoustic Code (TRAC) Prediction and Comparison with Measurements," American Helicopter Society 52nd Annual Forum, Washington D.C., June, 1996.

[8] Yu, Y. H., Caradonna, F. X., and Schmitz, F. H., "The Influence of the Transonic Flow Field on High-Speed Helicopter Impulsive Noise," Fourth European Rotorcraft and Powered Lift Aircraft Forum, Paper 58, 1978.
[9] Hanson, D. B. and Fink, M. R., "The Importance of Quadrupole Sources in Prediction of Transonic Tip Speed Propeller Noise," Journal of Sound and Vibration, Vol. 62, 1979, pp. 19-38.

[10] Brentner, K. S. and Holland, P. C., "An Efficient and Robust Method for Computing Quadrupole Noise," American Helicopter Society 1995 Aeromechanics Specialist Conference, Bridgeport, CT, 1995.

[11] Brentner, K. S., "An Efficient and Robust Method for Predicting Rotor High-Speed Impulsive Noise," AIAA paper 96-0151, 1996.

[12] Brentner, K. S., "Prediction of Helicopter Rotor Noise-A Computer Program Incorporating Realistic Blade Motions and Advanced Formulation," NASA TM-87721, 1986.

[13] Farassat, F., and Succi, G. P., "The Prediction of Helicopter Discrete Frequency Noise," Vertica, Vol. 7, No. 4, 1983, pp. 309-320.

[14] Lyrintzis, A. S., "Review: The Use of Kirchhoff's Method in Computational Aeroacoustics," ASME Journal of Fluids Engineering, Vol. 116, Dec. 1994, pp. 665-675.

[15] Farassat, F., and Myers, M. K., "Extension of Kirchhoff's Formula to Radiation from Moving Surfaces," Journal of Sound and Vibration, Vol. 123, No. 3, 1988, pp. 451-461.

[16] Farassat, F., and Myers, M. K., "The Kirchhoff formula for a Supersonically Moving Surface," CEAS/AIAA Paper 95-062 Proceedings of the 1st Joint CEAS/AIAA Aeroacoustics Conference (16th AIAA Aeroacoustics Conference), Vol. I, Munich, Germany, June 1995, pp. 455-463.

[17] Xue, Y., and Lyrintzis, A. S., "Rotating Kirchhoff Method for Three-Dimensional Transonic Blade-Vortex Interaction Hover Noise," AIAA Journal, Vol. 32, No. 7, Jul. 1994, pp. 1350-1359.

[18] Lyrintzis, A. S., Kilaras, M. S., and Xue, Y., "Transonic 3-D BVI Noise Using a Rotating Kirchhoff Formulation for Advancing Rotors," Proceedings of the 50th AHS Annual Forum, Vol. I, Washington, D.C., May 1994, pp. 115-127.

[19] Baeder, J. D., Gallman, J. M., and Yu, Y. H., "A Computational Study of Aeroacoustics of Rotors in Hover," American Helicopter Society 49th Annual Forum, St. Louis, MO, 1993. 
[20] Strawn, R. C., and Biswas, R., "Computation of Helicopter Rotor Noise in Forward Flight," Journal of the American Helicopter Society, Vol. 40, No. 3, July 1995, pp. 66-72.

[21] Strawn, R. C., Biswas, R., and Lyrintzis, A. S., "Helicopter Noise Predictions using Kirchhoff Methods," Proceedings of the 51st AHS Annual Forum, Vol. I, Fort Worth, Texas, May 1995, pp. 495-508. (To appear in Journal of Computational Acoustics.)

[22] Lyrintzis, A. S., Koutsavdis, E. K., Berezin, C., Visintainer, J., and Pollack, M., "Kirchhoff Acoustic Methodology Validation and Implementation to TiltRotor Aeroacoustic Codes (TRAC)," Proceedings of the 2nd American Helicopter Society International Specialists' Conference, Bridgeport, CT, Oct. 1995.

[23] Burley, C. L., and Tadghighi, H., "Importance of High Accuracy Blade Motion and Airloads Predictions in Acoustic Analysis," American Helicopter Society 50th Annual Forum, Washington, D. C., 1994.

[24] Purcell, T. W., "CFD and Transonic Helicopter Sound," Fourteenth European Rotorcraft Forum, Paper 2, 1988.

[25] Schmitz, F. H., Boxwell, D. A., Splettstoesser, W. R., and Schultz, K. J., "Model-Rotor HighSpeed Impulsive Noise: Full-Scale Comparisons and Parametric Variations," Vertica, Vol. 8, No. 4, 1984, pp. 395-422.

[26] Boxwell, D. A., Schmitz, F. H., Splettstoesser, W. R., and Schultz, K. J., "Model Helicopter Rotor High-Speed Impulsive Noise: Measured Acoustics and Blade Pressures," NASA TM-85850, Sept. 1983 .

[27] Yu, Y. H., Landgrebe, A. J., Liu, S. R., Lorber, P. F., Jordan, D. E., Pollack, M. J., and Martin, R. M., "Aerodynamic and Acoustic Test of a United Technologies Model Scale Rotor at DNW," Presented at 46th Annual Forum of the American Helicopter Society, Washington, D.C., May 2123,1990 .

[28] Liu, S. R., and Marcolini, M. A., "The Acoustic Results of a United Technologies Scale Model Helicopter Rotor Tested at DNW," Presented at the 46th Annual Forum of the American Helicopter Society, Washington, D.C., May 21-23, 1990. 